\title{
Geringe Samenbank von beweidbaren Arten für die Etablierung von Waldweiden im Schweizer Mittelland
}

\author{
Tabea Kipfer ${ }^{1,2}$ und Andreas Bosshard ${ }^{1,3}$ \\ ${ }^{1}$ Institut für Umweltwissenschaften, Universität Zürich, Winterthurerstrasse 190, CH-8057 \\ Zürich \\ 2 aktuelle Adresse: Eidg. Forschungsanstalt WSL, Zürcherstrasse 111, CH-8903 Birmensdorf; \\ e-mail: tabea.kipfer@wsl.ch \\ ${ }^{3}$ korrespondierender Autor: A. Bosshard, e-mail: abosshard@agraroekologie.ch
}

Manuskript angenommen am 2. September 2007

\begin{abstract}
Kipfer T. and Bosshard A. 2007. Low seed bank of herb species suitable for grazing hampers the establishment of wood pastures in the Swiss lowlands. Bot. Helv. 117:159167.

Controlled forest grazing is expected to yield benefits for biodiversity conservation, landscape quality, and in some cases also for land use economy. In the Swiss lowlands, first attempts are being made to reintroduce forest grazing in productive beech forests, but methodic experience is still limited. One main issue concerns the development of the vegetation after forest stands have been thinned to improve light conditions: Will grassland vegetation establish spontaneously? The present study analyses the composition of the soil seed bank of four beech forest stands. The seed bank density ranged from 1'244 to 28' 651 seeds $\mathrm{m}^{-2}$. Seed banks mainly consisted of forest and ruderal species; most abundant were Juncus effusus, Carex sylvatica, Rubus spp. and Clematis vitalba. Seeds of grassland plants were restricted to a few species, and their abundance in the seed bank decreased rapidly with increasing distance from the forest edge. These results reveal that there is little potential for grasslands to develop spontaneously from the seed bank. The introduction of grassland species of local origin using the green hay method is therefore recommended to prevent soil degradation during the first years of grazing, to fulfil minimal biodiversity requirements and to lower the risk of an establishment of neophytes and other problematic plant species.
\end{abstract}

Key words: Biodiversity, Fagetalia, forest grazing, seed input, vegetation development, forest soil. 


\section{Einleitung}

Waldweide war bis ins 19. Jahrhundert ein regulärer Bestandteil der Waldnutzung in Europa. Vielerorts führte das Fehlen von Regelungen zu Überweidung, welche die Verjüngung und die Schutzfunktion des Waldes beeinträchtigte (Grossmann 1927). Mit restriktiven Forstgesetzen wurde in der Schweiz und in anderen Ländern Europas die Waldweide seit Ende des 19. Jahrhunderts stark zurückgedrängt. Seit Jahrzehnten ist sie aus dem Schweizer Mittelland ganz verschwunden (Stuber und Bürgi 2001). Nur in höheren Lagen konnte sie sich regional als eigene Nutzungsform halten, z. B. die pâturages boisés (Wytweiden) im Jura (Gallandat und Gillet 1999; Perrenoud et al. 2003) und die Lärchenweiden in Graubünden und in den nordwestlichen Zentralalpen (Mayer et al. 2004).

Derzeit ist in Politik, Verwaltung, Naturschutz und bis hinein in Forstkreise ein wachsendes Interesse an der Waldweide festzustellen. Waldweidenutzung wird, sofern in geregelter Weise durchgeführt, als nachhaltige und auch wirtschaftlich und ökologisch attraktive Mehrnutzungsform des Waldes postuliert (Bürgi und Wohlgemuth 2002). Waldweiden schaffen für viele Pflanzen- und Tierarten wertvolle Lebensräume. Ein lichter, sorgfältig beweideter Wald bietet mehr und vor allem auch selteneren Tierund Pflanzenarten einen Lebensraum als die biomassereichen, zur Stammholzproduktion genutzten Waldformen wie Hoch- oder Plenterwälder. Ewald (2000) wies in einer breit angelegten Vergleichsstudie in beweideten Wäldern eine signifikant höhere Vielfalt geschützter und gefährdeter Pflanzenarten nach als in unbeweideten Referenzflächen. Hauptgründe für die hohe Biodiversität in Waldweiden sind das dauerhaft erhöhte Lichtangebot und der grosse Strukturreichtum, der durch die sich abwechselnden dichten und offenen, beweideten und unbeweideten Flächen entsteht (Schiess und Schiess-Bühler 1997). Die grösste Artenvielfalt an Pflanzen und Tieren findet man nicht im Innern der Wirtschaftswälder, sondern an den abgestuften und buchtigen Waldrändern und auf Waldlichtungen. Zahlreiche holzbewohnende Bock- und Prachtkäfer, Bienen-, Wanzen- und Fliegenarten sind auf Nektar und Pollen von Blütenpflanzen angewiesen, die nur auf Lichtungen und am Waldrand blühen (Forum Biodiversität Schweiz 2004). Fast alle Pflanzen- und Tierarten, die wir nur noch im extensiv genutzten Offenland, auf Magerwiesen, in Hochstammobstgärten usw. beobachten können, waren früher an vielen Stellen im Wald zu Hause (Schiess-Bühler und Schiess 1994). Mehrere Untersuchungen belegen einen Rückgang lichtbedürftiger Arten in den Wäldern des Mittellandes (Kuhn 1990; Egloff 1991; Walther und Grundmann 2001). Diesem negativen Trend könnte die geregelte Waldweide als Ergänzung zu den heute üblichen Naturschutzmassnahmen im Wald, aber auch zu einer lediglich holzorientierten Nutzung, entgegenwirken.

An verschiedenen Orten der Schweiz werden zurzeit Waldweide-Pionierprojekte geplant und durchgeführt. Zahlreiche Fragen sind aber noch unbeantwortet, von rechtlichen, praktischen, biologischen, edaphischen bis hin zu forstkundlichen (Schädigung des Zuwachses etc.). Wissenschaftliche Studien sind rar und fehlen insbesondere für Buchenstandorte, die im Mittelland häufigsten Waldstandorte (Schmid und Wiedemeier 2001). Eine der vordringlichen Fragen betrifft die Entwicklung der Pflanzendecke nach Auflichtung und Beweidung eines zuvor über viele Jahrzehnte geschlossenen Hochwaldes. Bildet sich relativ rasch eine geschlossene, beweidbare Pflanzendecke mit standortangepasster Pflanzenvielfalt, wie sie für eine bodenschonende Nutzung ebenso wie als Nahrungsbasis für das Vieh und die angestrebte Biodiversität wichtig ist? Welche Arten stellen sich von selbst ein und welche typischen 
Waldweidearten müssen gegebenenfalls eingebracht werden? Ziel der vorliegenden Studie war es, die Samenbank auf vier mehr oder weniger wüchsigen, typischen Buchenwaldstandorten des Schweizer Mittellandes zu untersuchen, und damit einen Beitrag zur nach wie vor geringen Kenntnis der Samenbank in Buchenwäldern des Mittellandes zu liefern.

\section{Methoden}

Ausgewählt für die Studie wurden vier Buchenwaldstandorte im Kanton Aargau. Zwei davon sind trockene Kalk-Buchenwälder auf flachgründigen Kalk-Rendzinen im südlichen Jura, und zwei weitere sind mittlere bis feuchte Waldmeister-Buchenwälder auf mässig sauren Braunerden im Reusstal (Tab. 1). An jedem Standort wurden 8-12

Tab. 1. Angaben zu den vier Untersuchungsgebieten: Politische Gemeinde, Meereshöhe (m ü.M.), Exposition, Mittlere Hangneigung (\%), im Gebiet vorkommende Waldgesellschaften und Anzahl untersuchter $5 \times 6 \mathrm{~m}^{2}$-Flächen $(n)$.

\begin{tabular}{|c|c|c|c|c|c|c|}
\hline $\begin{array}{l}\text { Name } \\
\text { Gemeinde }\end{array}$ & m ü.M. & Exp. & Neigung & $\begin{array}{l}\text { Waldgesell- } \\
\text { schaften* }\end{array}$ & Bodentyp & $n$ \\
\hline $\begin{array}{l}\text { Martinsberg } \\
\text { Baden }\end{array}$ & $405-500$ & SW & $65 \%$ & $9 \mathrm{a}, 10 \mathrm{a}, 13 \mathrm{~g}, 14 \mathrm{a}$ & $\begin{array}{l}\text { flachgründige } \\
\text { Kalk-Rendzina }\end{array}$ & 12 \\
\hline $\begin{array}{l}\text { Geissberg } \\
\text { Ennetbaden }\end{array}$ & $470-570$ & $\mathrm{~S}$ & $56 \%$ & $10 \mathrm{a}, 14 \mathrm{a}$ & $\begin{array}{l}\text { flachgründige } \\
\text { Kalk-Rendzina }\end{array}$ & 8 \\
\hline $\begin{array}{l}\text { Litzibuch N } \\
\text { Oberwil-Lieli }\end{array}$ & $565-600$ & $\mathrm{~N}$ & $35 \%$ & $7 \mathrm{a}, 7 \mathrm{aS}$ & $\begin{array}{l}\text { Braunerde, } \\
\text { saure Braunerde }\end{array}$ & 12 \\
\hline $\begin{array}{l}\text { Litzibuch S } \\
\text { Oberwil-Lieli }\end{array}$ & $585-610$ & NW & $15 \%$ & $7 a$ & $\begin{array}{l}\text { Braunerde, } \\
\text { saure Braunerde, Buntgley }\end{array}$ & 8 \\
\hline
\end{tabular}

* Nummerierung nach Stocker et al. (2002): 7a Typischer Waldmeister-Buchenwald; 7aS Typischer Waldmeister-Buchenwald mit Wald-Ziest; 9a Typischer Lungenkraut-Buchenwald; 10a Lungenkraut Buchenwald mit Immenblatt; $13 \mathrm{~g}$ Linden-Zahnwurz-Buchenwald mit Bärlauch; 14a Typischer Weissseggen-Buchenwald

Aufnahmeflächen $\left(5 \times 6 \mathrm{~m}^{2}\right)$ auf einem Raster (5-6 ha) zufällig ausgewählt und im Gelände eingemessen. Auf jeder Aufnahmefläche wurden Vegetationsaufnahmen nach Braun-Blanquet (1964) durchgeführt und Bodenproben für die SamenbankAnalyse gesammelt. Die Bodenproben umfassten ein Volumen von 21 und wurden wo dies möglich war - bis in eine Tiefe von $10 \mathrm{~cm}$ genommen. Sie wurden umgehend ins Gewächshaus gebracht und in Saatschalen auf einer Vermiculit-Unterlage ausgebreitet.

Während der Expositionszeit vom 27. Mai bis 26. Dezember 2005 wurden die auflaufenden Keimlinge regelmässig bestimmt, gezählt und dann aus den Saatschalen entfernt. Um die Keimung aller Samen zu fördern, wurde die Erde einmal ganz umgewühlt. Einige Vertreter der Gattungen Campanula, Carex, Cirsium, Luzula, Poa, Rubus, Tilia, Verbascum und Veronica sowie ein Individuum aus der Familie der Brassicaceae konnten lediglich bis auf die Gattung bzw. die Familie bestimmt werden. Um den Einflug von Fremdsamen zu verhindern, wurden die Saatschalen durch ein feinmaschiges Netz abgeschirmt. Zusätzlich wurden Kontroll-Saatschalen mit samenfreier Erde aufgestellt. Lediglich von Betula pubescens und Salix alba keimte je ein 
Individuum in den Kontrollschalen. Diese beiden Arten wurden deshalb von der Artenliste gestrichen.

Für die Auswertung wurden die Arten in folgende Gruppen gemäss ihren Ansprüchen an den Lebensraum eingeteilt: Äcker und Grünland, Wald (geschlossener Hochwald), Ruderalstellen (Schuttplätze, Wegränder, Ödland, Kiesgruben, etc.) und Saumstandorte (Waldränder, Säume, offene Stellen innerhalb von Wäldern, Waldschläge, Gehölze, Gebüsche). Für die Einteilung wurden die Standortangaben nach Lauber und Wagner (2001) verwendet.

\section{Ergebnisse}

Insgesamt wurden bei der Samenbank-Analyse 1'915 Keimlinge aus 75 Taxa (Art bzw. Gattung) erfasst (Anhang 1). 1'385 Keimlinge (72\% aller Keimlinge) gehörten zu den vier häufigsten Arten Carex sylvatica, Clematis vitalba, Juncus effusus und Rubus spp. $29 \%$ aller Arten waren mit nur einem Keimling belegt, weitere $13 \%$ mit zwei Keimlingen. Die Dichte der Samenbank variierte zwischen 1'244 (Geissberg) und 28'651 (Litzibuch Süd) Samen pro $\mathrm{m}^{2}$ Probefläche. Die hohen Zahlen für Litzibuch gehen vor allem auf Juncus effusus zurück (Tab. 2). Zwischen 40 und $50 \%$ der Arten in

Tab. 2. Durchschnittliche Samendichte und Artzusammensetzung der Samenbank.

\begin{tabular}{lcccccc}
\hline Gebiet & $\begin{array}{l}\text { Totale } \\
\text { Dichte } \\
\left(\mathrm{m}^{-2}\right)\end{array}$ & \multicolumn{6}{l}{\begin{tabular}{l} 
Anteil der Arten (\% der Keimlinge) \\
\cline { 3 - 6 }
\end{tabular}} & $\begin{array}{l}\text { Juncus } \\
\text { effus }\end{array}$ & $\begin{array}{l}\text { Carex } \\
\text { sylvatica }\end{array}$ & $\begin{array}{l}\text { Rubus } \\
\text { spp. }\end{array}$ & $\begin{array}{l}\text { Clematis } \\
\text { vitalba }\end{array}$ & andere \\
\hline Martinsberg & $4^{\prime} 311$ & 0 & 0 & 35 & 35 & 64 \\
Geissberg & $1^{\prime} 244$ & 0 & 0 & 16 & 16 & 84 \\
Litzibuch N & $7^{\prime} 511$ & 21 & 11 & 1 & 1 & 44 \\
Litzibuch S & $28^{\prime} 651$ & 75 & 10 & 0 & 0 & 11 \\
\hline
\end{tabular}

der Samenbank waren typische Waldarten, der Rest der Arten verteilte sich auf die drei Kategorien Ruderalarten, Arten von Saumstandorten und Arten von Äckern und Grünland (Abb. 1). Zudem fanden sich in der Samenbank auch zwei Neophyten (sensu SKEW 2004): Solidago gigantea (Martinsberg und Geissberg) und Buddleja davidii (Martinsberg).

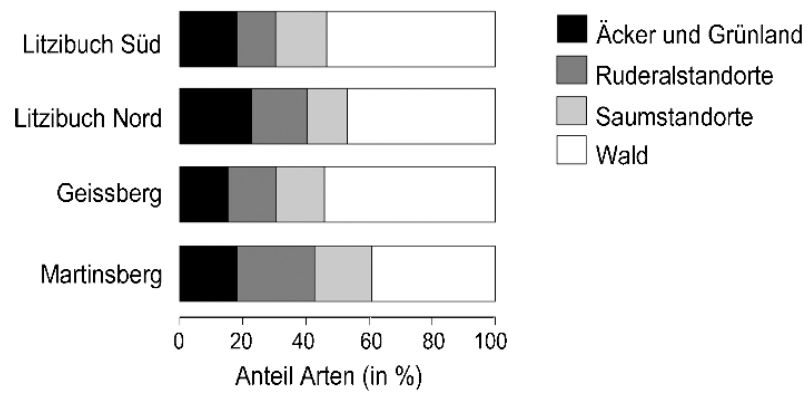

Abb. 1. Arten in der Samenbank eingeteilt nach Ansprüchen an den Lebensraum. 
Die Übereinstimmung zwischen aktueller Vegetation und Samenbank war klein. Während nur 2 bis $8 \%$ der Arten ausschliesslich in der Samenbank vorhanden waren, fanden sich mit 74 bis $88 \%$ der Arten die meisten Pflanzen nur in der aktuellen Vegetation. 7 bis $22 \%$ der Arten wurden sowohl in der Samenbank wie in der aktuellen Vegetation gefunden (Abb. 2). Diejenigen Arten, die in der aktuellen Vegetation dominierten, kamen nur teilweise auch in der Samenbank vor. Es waren dies Carex sylvatica, Clematis vitalba, Rubus spp. und Urtica dioica. Alle dominanten Holzpflanzen, die knapp die Hälfte der dominanten Arten in den Vegetationsaufnahmen ausmachten, fehlten in der Samenbank.

Der Anteil Arten von Äckern, Grünland und Saumstandorten in der Samenbank nahm mit zunehmender Distanz zum Waldrand signifikant ab (lineare Regression, $\mathrm{p}=0.004$ ) und entsprechend nahm der Anteil typischer Waldarten zu (lineare Regression, $\mathrm{p}=0.040 ;$ Abb. 3a und $\mathrm{b}$ ).

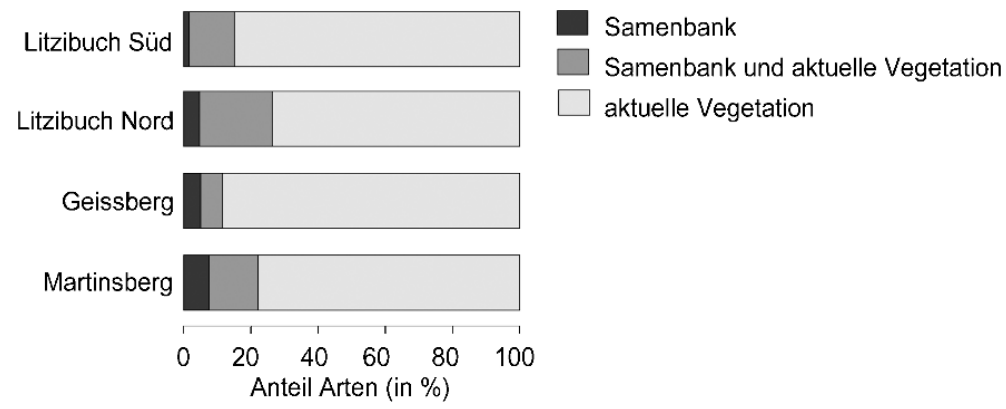

Abb. 2. Vergleich der Artenzusammensetzung von Samenbank und aktueller Vegetation.
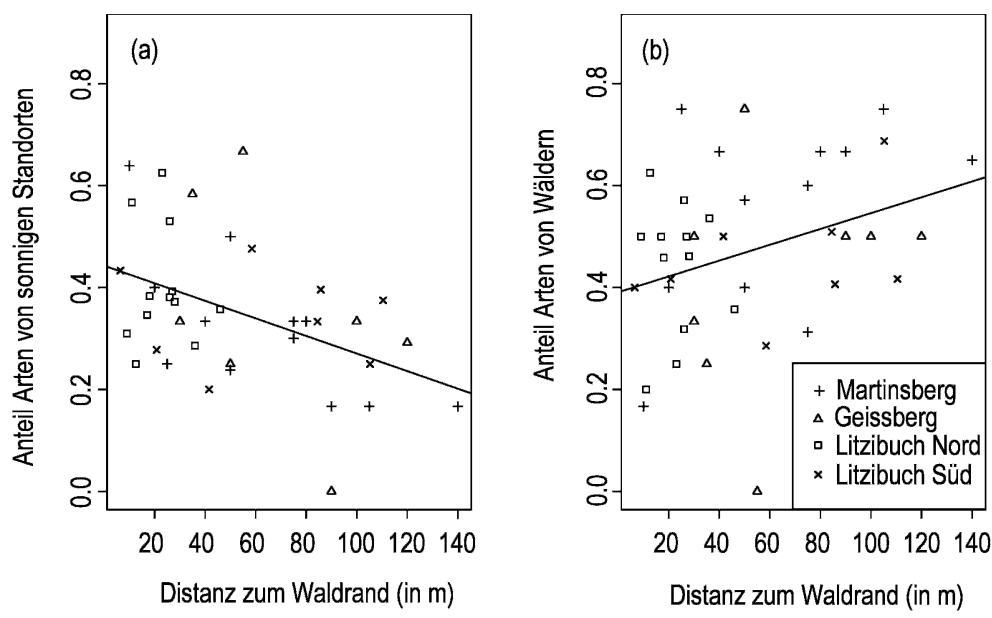

Abb. 3. Zusammensetzung der Samenbank in Abhängigkeit von der Distanz zum Waldrand: (a) Arten von sonnigen Standorten (Äcker, Grünland, Saumstandorte; $\mathrm{R}^{2}=0.19, p=0.004$ ),

(b) Waldarten $\left(\mathrm{R}^{2}=0.11, p=0.04\right)$. 


\section{Diskussion}

Die vorliegenden Resultate bestätigten für die hier untersuchten Buchenwälder zwei Feststellungen, wie sie für andere Waldtypen der gemässigten Zone in ähnlicher Weise gemacht wurden: Die Samenbank widerspiegelt nur zu einem sehr geringen Teil die in der aktuellen Vegetation auftretenden Arten (Abb. 2), und die Artenzahlen in der Samenbank sind gering. Lediglich zwischen 2 und 8\% der Arten kamen ausschliesslich in der Samenbank vor. Verglichen mit anderen Studien ist dieser Anteil sogar noch geringer. Decocq et al. (2004) fanden in einer Untersuchung in mehreren Eichen-Hagebuchenwäldern, dass $14 \%$ aller Arten nur in der Samenbank vorkommen. Bei der Studie von Ludemann (1994) in Tannen-Fichten-Beständen waren es 15\%. Pickett und McDonnell (1989) betrachten eine geringe Übereinstimmung der Samenbank mit der umgebenden Vegetation als generelles Merkmal von Samenbanken in Laubwäldern der gemässigten Zone.

Von den Arten, die in der aktuellen Vegetation dominierten, waren nur Carex sylvatica, Clematis vitalba, Rubus spp. und Urtica dioica in der Samenbank vertreten, die ersten drei Arten aber in grosser Anzahl (Tab. 2). Zusammen mit Juncus effusus machten sie den grössten Teil der Samenbank aus. Dies ist nicht aussergewöhnlich, zählen doch Rubus, Carex und Juncus zu den am häufigsten und oft auch in grossen Dichten gefundenen Gattungen in Samenbank-Untersuchungen (Pickett und McDonnell 1989).

Die Zusammensetzung der Samenbank ist auch vom Umfeld abhängig: Der Anteil der Arten, die typisch sind für Äcker, Grünland und Saumstandorte, nahm mit der Distanz zum jeweils nächsten Waldrand ab, während der Anteil typischer Waldarten entsprechend zunahm (Abb. 3a und b). Eine Extrapolation der Regressionskurve lässt vermuten, dass ab $250 \mathrm{~m}$ im Waldesinneren kein wesentlicher Sameneintrag von sonnigen Standorten ausserhalb des Waldes (Äcker, Grünland, Säume) mehr zu erwarten ist.

\section{Folgerungen für die Praxis}

Werden Buchenwaldstandorte aufgelichtet mit dem Ziel der Beweidung, fehlen in der Samenbank die Arten, welche eine stabile Grasnarbe bilden könnten, grösstenteils, oder sie kommen nur in kleiner Individuenzahl vor. Buchenwälder lassen im Gegensatz $\mathrm{zu}$ weniger wüchsigen und damit weniger dichten Waldtypen kaum Raum für mit Grünlandarten bewachsene Stellen. Die Arten müssen deshalb aus dem umgebenden Grünland einwandern. Dieser Prozess findet allerdings nur langsam statt, zumal nicht alle eintreffenden Diasporen in der Buchenlaubstreu günstige Etablierungsbedingungen finden. Zudem überleben die Samen der meisten Grünlandarten nur wenige Jahre in der Samenbank (Bakker et al. 1991; Davies und Waite 1998; Thompson et al. 1997). Die vorliegende Untersuchung zeigt auch, dass die Grünlandarten in der Samenbank im Waldesinneren bereits nach wenigen hundert Metern Entfernung zum Waldrand fehlen. Zudem sind viele der für eine Waldweide geeigneten und erwünschten Grünlandarten wie Festuca rubra, Bromus erectus, Trifolium pratense oder Centaurea jacea meist auch im umliegenden, in der Regel intensiv bewirtschafteten Wies- und Weideland nicht mehr vorhanden (Herzog und Walter 2005). Es ist deshalb damit zu rechnen, dass es Jahrzehnte dauert, bis sich eine an die Beweidung angepasste, 
stabile Vegetation einstellt, oder dass diese infolge grossräumigen Fehlens der betreffenden Arten gar nicht mehr entstehen kann.

Eine derart langsame Entwicklung einer beweidbaren Vegetation ist erstens aus Gründen des Bodenschutzes als problematisch zu beurteilen. Die in der Anfangsphase dominierenden Schlagflora- und Ruderalarten sind nicht an eine Beweidung angepasst. Sofern sie überhaupt gefressen werden, hinterlassen sie nach einer Abweidung einen kahlen Boden, weil sie kein Bestockungsvermögen aufweisen wie viele typische Weidepflanzen. Ohne Arten, die eine stabile Grasnarbe bilden, ist der Boden ungeschützt und empfindlich auf Bodenverdichtung und Erosion. Dies dürfte bei schweren Weidetieren, bei schlechter Witterung, bei Standweiden und auf steileren Flächen zu Problemen führen.

Der längerfristig offene Boden in einem aufgelichteten Bestand birgt zweitens auch die Gefahr, dass sich unerwünschte, später nur schwierig wieder zurückzudrängende Arten wie Pteridium aquilinum, Clematis vitalba, Juncus effusus und Rubus spp. etablieren können. Diese Arten bildeten mit Ausnahme von Pteridium aquilinum (nicht untersucht) einen sehr hohen Anteil an der Samenbank der untersuchten Flächen. Auch invasive Neophyten wie beispielsweise Solidago gigantea finden so geeignete Ausbreitungsbedingungen vor (Weber und Jakobs 2005). Unter den gegebenen Voraussetzungen ist es also unwahrscheinlich, dass sich eine artenreiche Vegetation einstellen kann - ein aus Naturschutzsicht wichtiges Ziel.

Die Resultate der vorliegenden Untersuchung legen nahe, dass für die Etablierung einer Waldweide geeignete Pflanzenarten eingebracht werden. Nur so kann sich in geeigneter Frist ein stabiler, standortgemässer und artenreicher Waldweidewasen bilden, welcher sowohl den Anforderungen einer Beweidung, des Bodenschutzes wie auch Biodiversitätszielen gerecht wird. Um eine genetische Florenverfälschung oder das Einbringen standörtlich und klimatisch nicht angepasster Pflanzen zu verhindern (Keller und Kollmann 1999), sollten nur regionale oder lokale Ökotypen verwendet werden. Diesen Anspruch erfüllt die Heugrassaat (Schnittgut-Diasporenübertragung), bei welcher artenreiche, standörtlich mit der Zielfläche vergleichbare Wiesen in der näheren Umgebung beerntet werden. Die Methode wurde für ähnliche Problemstellungen bereits mit Erfolg angewendet, so z. B. für die Wiederherstellung von artenreichen Wiesen auf landwirtschaftlich genutzten Flächen (Bosshard 1999; Hölzel und Otte 2003; Kiehl et al. 2006).

Besonders danken möchten wir der Fachstelle Naturschutz des Kantons Aargau und ihrem Leiter, André Stapfer, für die fachlichen Anregungen sowie für die finanzielle Unterstützung. Georg Schoop, Leiter Abteilung Stadtökologie der Stadt Baden, danken wir für sein grosses Engagement für die Waldweide-Projekte in Baden, in deren Rahmen ein Teil der vorliegenden Untersuchung durchgeführt werden konnte. Ebenso möchten wir Bernhard Schmid vom Institut für Umweltwissenschaften (Universität Zürich) und Rolf Rutishauser vom Institut für Systematische Botanik (Universität Zürich) danken für ihre fachlichen Hilfestellungen bei der Betreuung der Masterarbeit von T. Kipfer.

\section{Literatur}

Bakker J.P., Bos A.F., Hoogveld J. and Muller H.J. 1991. The role of the seed bank in restoration management of semi-natural grasslands. In: Ravera O. (ed.): Terrestrial and aquatic ecosystems: perturbation and recovery. Ellis Horwood, New York, 449-455.

Bosshard A. 1999. Renaturierung artenreicher Wiesen auf nährstoffreichen Böden. Ein Beitrag zur Optimierung der ökologischen Aufwertung der Kulturlandschaft und zum Ver- 
ständnis mesischer Wiesen-Ökosysteme. Diss. Bot. 303, Stuttgart. Online-version: http:// e-collection.ethbib.ethz.ch/show?type $=$ diss \&nr $=12922$.

Braun-Blanquet J. 1964. Pflanzensoziologie. Grundzüge der Vegetationskunde, 3. Aufl. Springer, Wien.

Bürgi M. und Wohlgemuth T. 2002. „Natur aus Bauernhand“ - auch im Wald? Inf.bl. Forsch.bereiches Landsch. 55. WSL, Birmensdorf.

Davies A. and Waite S. 1998. The persistence of calcareous grassland species in the soil seed bank under developing and established scrub. Plant Ecol. 136: 27-39.

Decocq G., Valentin B., Toussaint B., Hendoux F., Saguez R. and Bardat J. 2004. Soil seed bank composition and diversity in a managed temperate deciduous forest. Biodivers. Conserv. 13: 2485-2509.

Egloff F. 1991. Dauer und Wandel der Lägernflora. Vierteljahrsschr. Nat.forsch. Ges. Zür. 136: 207-270.

Ewald J. 2000. Long-term impact of forest pasture on the understorey of mountain forests in the Tegernsee Alps (Bavaria). Z. Ökol. Nat.schutz 9: 161-170.

Forum Biodiversität Schweiz (Hrsg.) 2004. Biodiversität in der Schweiz. Zustand - Erhaltung Perspektiven. Haupt, Bern.

Gallandat J.D. et Gillet F. 1999. Le pâturage boisé jurassien. Bull. Soc. Neuchâtel. Sci. Nat. 122: $5-25$.

Grossmann H. 1927. Die Waldweide in der Schweiz. Dissertation ETH Zürich.

Herzog F. und Walter T. (Hrsg.) 2005. Evaluation der Ökomassnahmen - Bereich Biodiversität. Schriftenreihe der FAL 56.

Hölzel N. and Otte A. 2003: Restoration of a species-rich flood meadow by topsoil removal and diaspore transfer with plant material. Appl. Veg. Sci. 6: 131-140.

Keller M. and Kollmann J. 1999. Effect of seed provenance on germination of herbs for agricultural compensation sites. Agric. Ecosyst. Environ. 72: 87-99.

Kiehl K., Thormann A. and Pfadenhauer J. 2006. Evaluation of initial restoration measures during the restoration of calcareous grasslands on former arable fields. Restor. Ecol. 14: $148-156$.

Kuhn N. 1990. Veränderung von Waldstandorten. Ber. Eidgenöss. Anst. forstl. Vers.wes. Nr. 319.

Lauber K. und Wagner G. 2001. Flora Helvetica, 3. Aufl. Haupt, Bern.

Ludemann T. 1994. Zum Samenvorrat von Waldböden im Feldberggebiet. Carolinea 52: $45-$ 60.

Mayer A.C., Stöckli V., Gotsch N., Konold W. und Kreuzer M. 2004. Waldweide im Alpenraum. Neubewertung einer traditionellen Mehrfachnutzung. Schweiz. Z. Forstwes. 155: $38-44$.

Perrenoud A., Känzig-Schoch U., Schneider O. und Wettstein J.-B. 2003. Nachhaltige Bewirtschaftung von Wytweiden. Ein Fallbeispiel aus dem Schweizer Jura. Haupt, Bern.

Pickett S.T.A. and McDonnell M.J. 1989. Seed bank dynamics in temperate deciduous forest. In: Leck M.A., Parker V.T. and Simpson R.L. (Eds). Ecology of soil seed banks. Academic Press, San Diego, 123-147.

Schiess-Bühler C. und Schiess H. 1994. Die frühere Waldnutzung hat es doch in sich. Natur und Mensch 1/1994: 19-22.

Schiess H. und Schiess-Bühler C. 1997. Dominanzminderung als ökologisches Prinzip: eine Neubewertung der ursprünglichen Waldnutzungen für den Arten- und Biotopschutz am Beispiel der Tagfalterfauna eines Auenwaldes in der Nordschweiz. Mitt. Eidgenöss. Forsch.anst. Wald Schnee Landsch. 72: 3-127.

Schmid W. und Wiedemeier P. 2001. Synthesebericht Weideliteratur. Agrofutura, Frick und Sternenberg, im Auftrag der Fachstelle Naturschutz des Kt. Zürich.

SKEW 2004. Schwarze Liste invasiver Neophyten. http://www.cps-skew.ch/deutsch/schwarze_liste.htm (Stand 20.4.07). 
Stocker R., Burger T., Elsener O., Liechti T., Portmann-Orlowski K. und Zantop S. 2002. Die Waldstandorte des Kantons Aargau. Hrsg. vom Finanzdepartement des Kt. Aargau, Abt. Wald, Aarau.

Stuber M. und Bürgi M. 2001. Agrarische Waldnutzungen in der Schweiz: 1800-1950. Waldweide, Waldheu, Nadel- und Laubfutter. Schweiz. Z. Forstwes. 152: 490-508.

Thompson K., Bakker J. and Bekker R. 1997: The soil seed banks of North West Europe: methodology, density and longevity. Cambridge University Press, Cambridge.

Walther G.-R. and Grundmann A. 2001. Trends of vegetation change in colline and submontane climax forests in Switzerland. Bull. Geobot. Inst. ETH 67: 3-12.

Weber E. and Jakobs G. 2005. Biological flora of central Europe: Solidago gigantea Aiton. Flora 200: $109-118$.

Anhang

Anhang 1. Zusammensetzung der Samenbank der 40 Probeflächen.

Dieser Anhang kann kostenlos von http://www.birkhauser.ch/BH heruntergeladen werden: (unter "Additional Informations" auf "Electronic Supplementary Material" klicken). 